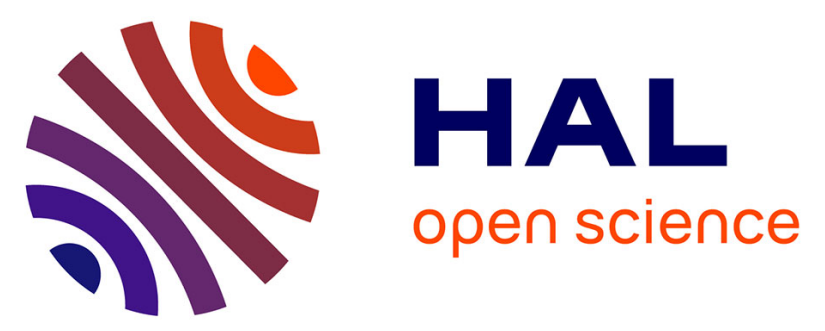

\title{
RDFI determination of anisotropic and scattering dependent radiative conductivity tensors in porous media: Application to rod bundles
}

Fabien Bellet, Elie Chalopin, Florian Fichot, Estelle Iacona, J. Taine

\section{- To cite this version:}

Fabien Bellet, Elie Chalopin, Florian Fichot, Estelle Iacona, J. Taine. RDFI determination of anisotropic and scattering dependent radiative conductivity tensors in porous media: Application to rod bundles. International Journal of Heat and Mass Transfer, 2009, 52 (5-6), pp.1544-1551. 10.1016/j.ijheatmasstransfer.2008.08.007 . hal-00340094

\section{HAL Id: hal-00340094 \\ https://hal.science/hal-00340094}

Submitted on 6 Oct 2011

HAL is a multi-disciplinary open access archive for the deposit and dissemination of scientific research documents, whether they are published or not. The documents may come from teaching and research institutions in France or abroad, or from public or private research centers.
L'archive ouverte pluridisciplinaire HAL, est destinée au dépôt et à la diffusion de documents scientifiques de niveau recherche, publiés ou non, émanant des établissements d'enseignement et de recherche français ou étrangers, des laboratoires publics ou privés. 


\title{
RDFI determination of anisotropic and scattering dependent radiative conductivity tensors in porous media. Application to rod bundles.
}

\author{
Fabien Bellet ${ }^{\mathrm{a}}$, Elie Chalopin ${ }^{\mathrm{b}}$, Florian Fichot ${ }^{\mathrm{b}}$, \\ Estelle Iacona $^{\mathrm{a}, *}$, Jean Taine ${ }^{\mathrm{a}}$ \\ ${ }^{a}$ Laboratoire EM2C, École Centrale Paris - UPR 288 CNRS, \\ 92295 Châtenay-Malabry Cedex, France \\ ${ }^{\mathrm{b}}$ Institut de Radioprotection et de Sûreté Nucléaire, site de Cadarache \\ BP 3, 13115 Saint-Paul-Lez-Durance Cedex, France
}

\begin{abstract}
A radiative conductivity model is developed for porous media with a solid opaque phase and a transparent fluid phase. In a first step, an effective semi transparent medium occupying the volume of the real fluid phase is characterized, assuming the validity of the Beer's laws. For example, rod bundles in squared or triangular configurations can be directly characterized by effective strongly anisotropic extinction, absorption and scattering coefficients, optical index and phase function, which depends on both the incident and scattering unit vectors, by generalizing the Radiative Distribution Function Identification method of Tancrez and Taine (2004). The validity and accuracy of the associated Beer's laws are discussed in this case. In a second step, at the limit of an optically thick porous medium, an original model based on a perturbation method of the Radiative Transfer Equation directly leads to the determination, under an accurate validity criterion, of a radiative conductivity tensor for the fluid phase. Examples of results are given in the case of rod bundles versus porosity, specific area and local wall absorptivity.
\end{abstract}

Key words: Radiative properties, porous medium, radiation scattering, perturbation method, radiative conductivity, rod bundles

* Corresponding author. Tel.: +33141 1310 75; fax: +33 147028035 Email address: estelle.iacona@em2c.ecp.fr (Estelle Iacona). 


\section{Nomenclature}

Latin symbols

$A \quad$ Specific area per unit volume of the fluid phase

$\mathrm{Kn}_{\nu}^{a} \quad$ Radiative Knudsen number

u Unit vector

n Normal unit vector

$D \quad$ Rod diameter, $\mathrm{m}$

$G_{e} \quad$ Extinction cumulated distribution function

$g_{e} \quad$ Extinction cumulated distribution function for a semi transparent medium

I Impact point

$k \quad$ Radiative conductivity

$k_{i j} \quad$ Components of the radiative conductivity tensor, $\mathrm{W} / \mathrm{m} / \mathrm{K}$

$M \quad$ Point in the fluid phase

$n \quad$ Optical index

$P \quad$ Distance between the axes of two consecutive cylinders in a configuration independent direction, $\mathrm{m}$

$P_{a} \quad$ Absorption cumulated probability

$p_{a} \quad$ Absorption cumulated probability for a semi transparent medium

$s \quad$ Curvilinear abscissa along a ray

$T_{w} \quad$ Wall temperature, $\mathrm{K}$

$x_{i} \quad$ Coordinate of the axis i

Greek symbols

$\alpha \quad$ Wall absorptivity

$\beta \quad$ Extinction coefficient

$\kappa \quad$ Absorption coefficient

$\Omega \quad$ Solid angle

$\Pi \quad$ Porosity

$\sigma \quad$ Scattering coefficient

$\theta, \varphi \quad$ Euler angles

$\varepsilon_{X} \quad$ Identification error criterion for the parameter $X$

Indexes

$+\quad$ Non-dimensional

' Directional

(i) Order i of perturbation

diff Diffuse reflection law

$h \quad$ Hemispheric

spec Specular reflection law

$\nu \quad$ Frequency

a Absorption

e Extinction

$i \quad$ Direction i 


\section{Introduction}

An accurate modeling of radiative transfer is required in many applications involving porous media at high temperature. For these strongly heterogeneous media, the only continuous equivalent model today available in practice is an effective semi transparent medium model, characterized by the Beer's laws. The radiative properties of such an effective medium are generally been indirectly obtained by parameters identification techniques. The extinction and absorption coefficients and phase function are then characterized by parameters, which are identified by minimizing discrepancies between, on the one hand, generally experimental intensity, reflectance or transmittance results, and on the other hand, results of a global radiative transfer model applied, in the same conditions, to the effective medium. For different types of porous media, the works of Hendricks and Howell [1-3], Baillis and Sacadura [4] and Singh and Kaviany [5] are representative of this approach. A more detailed bibliography has been given by Tancrez and Taine [6] and Zeghondy et al. [7]. Due to the large number of parameters to determine, these methods very often lead to a result, while Beer's laws are assumed to be verified for the effective medium without any validity criterion.

Based on a statistical representation of a porous medium, the Radiation Distribution Function Identification (RDFI) method of Refs. [6] and [7] only requires the knowledge of the real medium morphology and radiative properties at a local scale. Indeed, a porous medium is completely characterized by an extinction cumulated distribution function, an absorption cumulated probability and a scattering phase function. The RDFI approch is based on the identification of the extinction cumulated distribution functions and absorption cumulated probabilities of the real porous medium and of an effective semi transparent medium. It also leads to a quantitative validity criterion of the Beer's laws, associated with these identifications. This method has been applied to statistically isotropic virtual media of high porosity, made of transparent (respectively opaque) spheres in an opaque (respectively transparent) solid phase [6], to a statistically anisotropic real mullite foam [7] and to a reticulated ceramic foam by Petrasch et al. [8].

For an optically thick porous medium, a radiative conductivity model is used in practice. In the case of rod bundles, Fischer [9], Cox [10], Tien et al. [11], Sohal [12], Chatelard [13], Manteufel [14] have calculated a radiative conductivity from a model at the local scale which generally requires many approximations (for instance, limitation to transfer with the closest rods). These authors often use simplified variants of the zonal method of Hottel and Sarofim [15]. Mazza et al. [16] and Rubiolo and Gatt [17] use a multi-layer approach. But, due to the required approximations, the predictions by different models $[9,13,14,17]$ of the rod bundle equivalent conductivity, in a typical configuration defined 
in Ref. [18], can differ by a factor up to 3 .

The present work deals with the determination, for a statistically strongly anisotropic porous medium with an opaque phase, of: i) the radiative properties of an effective semi transparent medium by the RDFI method in Sec. 2; ii) the associated radiative conductivity tensor, at the medium optically thick limit in Sec. 3. In both cases, validity criteria are introduced and the application example is a rod bundle, either in squared or triangular configuration for a large range of porosities and wall absorptivities.

\section{Radiative properties of the system}

This section deals with the characterization of the radiative properties of the effective medium associated with a Representative Elementary Volume, called REV1, of a parallel opaque rod bundle in a transparent fluid or in vacuum. At this step, no assumption is made on the REV1 optical thickness. In Sec.3 a larger REV, optically thick and called REV2, will be considered, in order to introduce the radiative conductivity tensor.

The effective properties considered here are those of an equivalent semi transparent medium which occupies the same volume as the fluid phase of porosity $\Pi$, but is characterized by the temperature $T_{w}$ of the rod opaque walls in the REV1. Physically, no radiative power is dissipated in the transparent phase. Two types of parallel opaque rod bundle configurations, characterized by elementary triangular and squared patterns in a cross section, are considered and given in Figs. 1(a) and 1(b). The system coordinates are defined in Fig. 1(c). The bundle porosity $\Pi$ is a function of $P / D$, ratio of the distance between the axes of two consecutive cylinders to their diameter

$$
\begin{aligned}
& \Pi=1-\frac{\pi}{2 \sqrt{3}(P / D)^{2}} \quad \text { (triangular pattern) } \\
& \Pi=1-\frac{\pi}{4(P / D)^{2}} \quad \text { (squared pattern). }
\end{aligned}
$$

The specific area per unit volume of the fluid phase, $A$, is the dimensional parameter associated with $D$. For both pattern, we have

$$
A=\frac{4(1-\Pi)}{\Pi D}
$$

At the local scale, the opaque rod absorption law is assumed to be either diffuse and characterized by a wall absorptivity $\alpha_{\nu}^{h}$, or approximately specular 
with the same hemispherical wall absorptivity $\alpha_{\nu}^{h}$

$$
\alpha_{\nu}^{\prime}(\mathbf{u}, \mathbf{n})=\frac{3}{2} \alpha_{\nu}^{h}(-\mathbf{u . n}) \quad ; \quad-\mathbf{u . n}=-\sin \theta \cos \left(\varphi-\varphi_{0}\right)=\mu \geq 0,
$$

where $\mathbf{u}(\theta, \varphi)$ is the direction of the incident ray and $\mathbf{n}\left(\varphi_{0}\right)$ the local normal to a rod, as defined in Fig. 1(c). The notation $\alpha_{\nu}^{\prime}\left(\theta, \varphi, \varphi_{0}\right)$ will sometimes be used in the following.

\subsection{Application of the Radiation Distribution Function Identification method}

The RDFI method, detailed in Refs. [6,7], is applied to the previous systems, which are both statistically anisotropic porous media.

\subsubsection{Extinction coefficient}

For a given solid angle $\mathrm{d} \Omega=\sin \theta \mathrm{d} \theta \mathrm{d} \varphi$, the extinction coefficient $\beta_{\nu}(\theta, \varphi)$ is obtained by identification of $G_{e}(s, \theta, \varphi)$, the extinction cumulated distribution function of the real medium, with the corresponding extinction cumulated distribution function of the equivalent semi transparent medium, given by

$$
g_{e}(s, \theta, \varphi)=1-\exp \left[-\beta_{\nu}(\theta, \varphi) s\right]
$$

As detailed in Ref. [7], $G_{e}(s, \theta, \varphi)$ is in fact the probability of extinction at an impact point $I$ from a current source point $M(\mathbf{r})$, before a distance $s$ and along a current ray of unit vector $\mathbf{u}$ belonging to an elementary angle $d \Omega$

$$
G_{e}(s, \theta, \varphi) \mathrm{d} \Omega=\int_{0}^{s} \frac{1}{V_{F}} \int_{V_{F}} \delta\left[s^{\prime}-s_{0}(\mathbf{r}, \theta, \varphi)\right] \mathrm{d} \mathbf{r} \mathrm{d} s^{\prime} \mathrm{d} \Omega,
$$

where $s_{0}(\mathbf{r}, \theta, \varphi)$ is the distance from $M(\mathbf{r})$, center of the volume element $\mathrm{d} \mathbf{r}$, to the impact point I, $\delta$ the Dirac distribution function and $V_{F}$ the volume of the fluid phase. $G_{e}(s, \theta, \varphi)$ only depends on the geometry and is then independent of the radiation frequency $\nu$. Consequently, $\beta(\theta, \varphi)$ does not depend on $\nu$. Moreover, due to the axial symmetry of the considered systems, we obtain

$$
s_{0}(\mathbf{r}, \theta, \varphi)=\frac{s_{0}(\mathbf{r}, \pi / 2, \varphi)}{\sin \theta}
$$


As demonstrated in Appendix 1, a consequence of Eqs. 6 and 7 is that

$$
G_{e}(s, \theta, \varphi)=G_{e}(s \sin \theta, \pi / 2, \varphi) .
$$

Due to the axial symmetry of the system, we obtain, from Eqs. 5 and 8

$$
\beta(\theta, \varphi)=\beta(\pi / 2, \varphi) \sin \theta
$$

As a result, we will only identify, in Sec. $2.2, G_{e}(s, \pi / 2, \varphi)$ with the corresponding extinction cumulated distribution function for the equivalent semi transparent medium, $g_{e}(s, \pi / 2, \varphi)$.

\subsubsection{Absorption coefficient}

Similarly, the absorption coefficient $\kappa_{\nu}(\theta, \varphi)$, dependent on $\nu$, is obtained by identification of the absorption cumulated probability of the real porous medium, $P_{a \nu}(s, \theta, \varphi)$, with the corresponding probability for the equivalent semi transparent medium $[6,7]$

$$
p_{a \nu}(s, \theta, \varphi)=\kappa_{\nu}(\theta, \varphi) / \beta(\theta, \varphi)(1-\exp [-\beta(\theta, \varphi) s]) .
$$

By generalizing Eq. 4 of Ref. [6], the absorption cumulated probability before a distance $s$, and along a current ray of unit vector $\mathbf{u}$ belonging to an elementary angle $\mathrm{d} \Omega$ is

$$
P_{a \nu}(s, \theta, \varphi) \mathrm{d} \Omega=\int_{0}^{s} \frac{1}{V_{F}} \int_{V_{F}} \alpha_{\nu}^{\prime}\left[\theta, \varphi, \varphi_{0}(\mathbf{r}, \varphi)\right] \delta\left[s^{\prime}-s_{0}(\mathbf{r}, \theta, \varphi)\right] \mathrm{d} \mathbf{r} \mathrm{d} s^{\prime} \mathrm{d} \Omega .(11)
$$

This equation only differs from Eq. 6 by the factor $\alpha_{\nu}^{\prime}\left[\theta, \varphi, \varphi_{0}\right]$ which represents the rod absorption probability. For a diffuse reflection law, since $P_{a \nu}^{\text {diff }}=$ $\alpha_{\nu}^{h} G_{e}^{\text {diff }}$, the absorption coefficient $\kappa_{\nu}^{\text {diff }}(\theta, \varphi)$ is simply given by

$$
\kappa_{\nu}^{\text {diff }}(\theta, \varphi)=\alpha_{\nu}^{h} \beta(\theta, \varphi) .
$$

In the case of a specular reflection law, as demonstrated in Appendix 1, a consequence of Eqs. 7 and 11 is that

$$
P_{a \nu}^{s p e c}(s, \theta, \varphi)=\sin \theta P_{a \nu}^{s p e c}(s \sin \theta, \pi / 2, \varphi) .
$$

Consequently, we obtain, from Eqs. 10 and 13

$$
\kappa_{\nu}^{\text {spec }}(\theta, \varphi)=\kappa_{\nu}^{\text {spec }}(\pi / 2, \varphi) \sin ^{2} \theta
$$


In this expression, a $\sin \theta$ comes as before from Eq. 7, and the second one from the proportionality of $\alpha_{\nu}^{\prime}\left[\theta, \varphi, \varphi_{0}\right]$ with $\sin \theta$ in Eq. 4 . As a result, we will only identify, in Sec. 2.2, $P_{a \nu}^{s p e c}(s, \pi / 2, \varphi)$ with the corresponding extinction cumulated probability for the effective semi transparent medium, $p_{a \nu}^{s p e c}(s, \pi / 2, \varphi)$.

\subsection{Results for extinction and absorption coefficients}

In order to define a reference for the calculations of $\beta(\pi / 2, \varphi)$, let us consider a global extinction coefficient $\beta_{O T}(\theta)$, independent of $\varphi$, at the medium optically thin limit and for a diffuse reflection law. In these conditions, the flux emitted by the interfacial area $\mathrm{d} S$ of a REV1 can be identified with the flux emitted by the associated volume of the effective medium fluid phase $\mathrm{d} V_{F}$. If we crudely assume the effective optical index equal to 1, and by using Eqs. 9 and 12, we obtain

$$
\pi \alpha_{\nu}^{h} I_{\nu}^{\circ}(T) \mathrm{d} S \mathrm{~d} \nu=\left(\int_{0}^{\pi} \alpha_{\nu}^{h} \beta_{O T}(\pi / 2) 2 \pi \sin ^{2} \theta \mathrm{d} \theta\right) I_{\nu}^{\circ}(T) \mathrm{d} V_{F} \mathrm{~d} \nu,
$$

from which we deduce that $\beta_{O T}(\pi / 2)$ is equal to $A / \pi$. The non dimensional extinction coefficient $\beta^{+}(\pi / 2, \varphi)$ is then defined by

$$
\beta^{+}(\pi / 2, \varphi)=\frac{\beta(\pi / 2, \varphi)}{\beta_{O T}(\pi / 2)}=\frac{\beta(\pi / 2, \varphi) \pi}{A}
$$

From dimensional analysis, $\beta(\pi / 2, \varphi)$ and consequently $\kappa_{\nu}^{\text {diff }}(\pi / 2, \varphi)$ are the product of $A$ by a function of $\Pi$. As a result, $\beta^{+}$does not depend on $A$. The non dimensional specular absorption coefficient $\kappa^{+\operatorname{spec}}(\pi / 2, \varphi)$, defined by

$$
\kappa^{+\operatorname{spec}}(\pi / 2, \varphi)=\kappa_{\nu}^{\text {spec }}(\pi / 2, \varphi) /\left[\alpha_{\nu}^{h} \beta(\pi / 2, \varphi)\right]
$$

is independent of $\nu$, because $\kappa_{\nu}^{\text {spec }}$ is proportional to $\alpha_{\nu}^{h}$. Furthermore, since the extinction length distribution is independent of the local reflection law, $\kappa_{\nu}^{\text {spec }}$ is the product of $A$ by a function of $\Pi$, and $\kappa^{+ \text {spec }}$ does not depend on A.

In the Monte Carlo technique, a large number of rays is generated from randomly chosen points $M$ within the fluid phase and into randomly chosen directions, which leads to huge sets of impact points I at the interface. For each ray, the value of the distance $M I$ obtained is a contribution to the extinction distribution function. In practice, as a consequence of Eqs. 9 and 14, the rays are only generated from points $M$ in a shooting area located in the cross section $\theta=\pi / 2$ of the system. This shooting area takes into account the system 
symmetries: it is either a regular hexagonal area limited by six cylinders and containing one cylinder in its center, as shown in Fig. 1(a), or a squared area limited by four cylinders, as shown in Fig. 1(b). The system exhibits a mirror symmetry with respect to $\pi / 6$ (triangular) or $\pi / 4$ (squared), and then a $\pi / 3$ (triangular) or $\pi / 2$ (squared) periodicity. As a consequence, the calculations have only been carried out for 30 values of $\varphi$ regularly spaced in the range $[0, \pi / 6]$ (triangular) or $[0, \pi / 4]$ (squared).

The RDFI method has been applied to squared and triangular bundle configurations for $\theta=\pi / 2$. For each discretized value of $\varphi_{k}, \beta^{+}\left(\pi / 2, \varphi_{k}\right)$ and $\kappa^{+\operatorname{spec}}\left(\pi / 2, \varphi_{k}\right)$, which allow us to calculate $\beta\left(\theta, \varphi_{k}\right), \kappa_{\nu}^{\text {diff }}\left(\theta, \varphi_{k}\right)$ and $\kappa_{\nu}^{\text {spec }}\left(\theta, \varphi_{k}\right)$, have been systematically calculated, for the squared (resp. triangular) pattern, and for 16 values of $\Pi$ (resp. 18 values of $\Pi$ ) between 0.2146 and 0.99 (resp. 0.093 and 0.99 ). The smallest values of $\Pi$ differ: indeed, the maximum compacity is higher for a triangular pattern than for a squared one.

The size of the calculation domain has been chosen in such a manner that, in the elementary solid angle $\varphi_{k}$ where $\beta\left(\pi / 2, \varphi_{k}\right)$ has the smallest value, $1-\exp (-3) \simeq 95 \%$ of the rays are extinguished. This size has been first evaluated for $10^{5}$ rays, shot in this solid angle. The convergence of the Monte Carlo method is characterized by a relative standard deviation between 10 sets of $10^{7}$ rays, which is here of the order of $6 \cdot 10^{-4}$. The typical relative difference between standard deviations calculated with $10^{8}$ and $10^{9}$ rays is less than some $10^{-4}$ for all porosities. Consequently, $10^{8}$ rays have been used for all calculations. The determinations of $G_{e}$ and $P_{a}$ is then very accurate.

As in Ref. [7], the $\beta\left(\pi / 2, \varphi_{k}\right)$ identification error criterion, $\varepsilon_{e}\left(\beta\left(\pi / 2, \varphi_{k}\right)\right)$, is

$$
\begin{aligned}
& \varepsilon_{e}\left(\beta\left(\pi / 2, \varphi_{k}\right)\right)= \\
& {\left[\sum_{i=0}^{N}\left[G_{e}\left(s_{i}, \pi / 2, \varphi_{k}\right)-g_{e}\left(s_{i}, \pi / 2, \varphi_{k}\right)\right]^{2} / \sum_{i=0}^{N}\left[1-G_{e}\left(s_{i}, \pi / 2, \varphi_{k}\right)\right]^{2}\right]^{1 / 2} .}
\end{aligned}
$$

An example of $G_{e}\left(s, \pi / 2, \varphi_{k}\right)$ is given in Fig. .2, for a triangular configuration and the porosity $\Pi=0.513$. A complete set of results is given in Ref. [18]. The function $G_{e}(s, \pi / 2)$, cumulated for rays associated with all $\varphi$ values in $[0, \pi / 6]$, is also plotted. The identification of $G_{e}(s, \pi / 2)$ with $g_{e}(s, \pi / 2)$ is accurate $\left(\varepsilon_{e}\left[\beta^{+}(\pi / 2)\right]=0.016\right)$, and leads to an extinction coefficient $\beta$. The Fig. .2 shows the identified extinction cumulated distribution function $g_{e}$, function of $\beta s$. It also appears in Fig. .2 that the shape of $G_{e}\left(s, \pi / 2, \varphi_{k}\right)$ can deviate from an exponential function, which is associated with the Beer's law. These results are quantified in Fig. 3(a), which shows identified values of $\beta^{+}(\pi / 2, \varphi)$ and the associated $\varepsilon_{e}\left[\beta^{+}(\pi / 2, \varphi)\right]$ versus $\varphi$ for different porosity values, including 0.513 . It appears that $\varepsilon_{e}\left[\beta^{+}(\pi / 2, \varphi)\right]$ becomes larger and larger when the porosity increases. For some singular directions corresponding to alleys between rods, $\varepsilon_{e}\left[\beta^{+} \beta\left(\pi / 2, \varphi_{k}\right)\right]$ can even take high values. As $G_{e}$ is always determined very accurately, it appears that the Beer's law is less and 
less verified when the porosity increases from 0.5 to 0.99 .

$\beta^{+}(\pi / 2)$, extracted from $G_{e}(s, \pi / 2)$, is plotted in Fig. 3(b). While values of $\varepsilon_{e}\left[\beta^{+}(\pi / 2, \varphi)\right]$ can be high, the typical value of $\varepsilon_{e}\left[\beta^{+}(\pi / 2)\right]$ is less than $2.410^{-2}$, due to some compensation between directions. It is a much more accurate result than in previous works, even for high porosity values. In the considered very anisotropic geometry, the accuracy on $\beta^{+}(\pi / 2)$ results is better for low values of $\Pi$ than in the case of the statistically isotropic media of Ref [6]. Indeed, the effect of the system infinite direction is weak for low values of $\Pi$, and on the contrary, it becomes large for higher porosities. Results for triangular and squared patterns are similar. Finally, the same conclusions can be drawn for $\kappa^{+\operatorname{spec}}(\pi / 2)$ with Fig. 3(c).

\subsection{Direct determination of the anisotropic phase function}

The phase function $p_{\nu}\left(\mathbf{u}, \mathbf{u}_{\mathbf{r}}\right)$ is defined by the fact that $p_{\nu}\left(\mathbf{u}, \mathbf{u}_{\mathbf{r}}\right)\left(\mathrm{d} \Omega_{r} / 4 \pi\right)$ is the probability that the intensity extinguished by scattering in the solid angle $\mathrm{d} \Omega(\theta, \varphi)$ is scattered in the solid angle $\mathrm{d} \Omega_{r}\left(\theta_{r}, \varphi_{r}\right) \cdot p_{\nu}\left(\mathbf{u}, \mathbf{u}_{\mathbf{r}}\right)$ is expressed from the bidirectional reflectivity $\rho_{\nu}^{\prime \prime}$ of the opaque wall, as in Ref. [6], by

$$
\begin{aligned}
& \frac{1}{4 \pi} p_{\nu}\left(\mathbf{u}, \mathbf{u}_{\mathbf{r}}\right) \mathrm{d} \Omega_{r} \\
= & \frac{1 / V_{F} \int_{V_{F} / \cos \left(\varphi_{0}(\mathbf{r}, \varphi)-\varphi_{r}\right) \geq 0} \rho_{\nu}^{\prime \prime}\left(\mathbf{u}, \mathbf{u}_{\mathbf{r}}, \mathbf{n}(\mathbf{r}, \varphi)\right)(-\mathbf{u . n}(\mathbf{r}, \varphi)) \mathrm{d} \mathbf{r} \mathrm{d} \Omega_{r}}{\int_{4 \pi} 1 / V_{F} \int_{V_{F} / \cos \left(\varphi_{0}(\mathbf{r}, \varphi)-\varphi_{r}^{\prime}\right) \geq 0} \rho_{\nu}^{\prime \prime}\left(\mathbf{u}, \mathbf{u}_{\mathbf{r}}^{\prime}, \mathbf{n}(\mathbf{r}, \varphi)\right)(-\mathbf{u} \cdot \mathbf{n}(\mathbf{r}, \varphi)) \mathrm{d} \mathbf{r} \mathrm{d} \Omega_{r}^{\prime}} .
\end{aligned}
$$

The summations in Eq. 19 are in practice carried out within the Monte Carlo calculations of Sec. 2.1. If we consider the global homogenized system, $\varphi$ and $\varphi_{r}$ independently belong to the range $[0,2 \pi]$. For a given couple $\left(\mathbf{u}, \mathbf{u}_{r}\right)$, the only shooting points $\mathbf{r}$ considered are those for which the reflected ray does not go through the rod, i.e. $\mathbf{u}_{\mathbf{r}} \cdot \mathbf{n} \geq 0$ or $\cos \left(\varphi_{0}(\mathbf{r}, \varphi)-\varphi_{r}\right) \geq 0$. Since $\varphi_{0}$ is the angle of the normal vector at the first impact point between the incident ray and the cylinder, the incident ray never enters in the rod: $\mathbf{u} . \mathbf{n} \leq 0$, i.e. $\cos \left(\varphi_{0}(\mathbf{r}, \varphi)-\varphi\right) \leq 0$. Indeed, at this local scale, and for a given impact, $\varphi_{0}-\varphi$ is in the range $[\pi / 2,3 \pi / 2]$. Note that $p_{\nu}$ does not depend on $A$ : for a given $\Pi$, a change in $A$ is an homothety ( $D$ changes but $P / D$ remains constant), which keeps angles unchanged.

For a diffuse reflection law, the local bidirectional reflectivity is uniform and equal to $\left(1-\alpha_{\nu}^{h}\right) / \pi$, and we obtain a phase function independent of $\theta$ and $\theta_{r}$

$$
p^{\text {diff }}\left(\varphi, \varphi_{r}\right)=2 \pi \frac{\int_{V_{F} / \cos \left(\varphi_{0}(\mathbf{r}, \varphi)-\varphi_{r}\right) \geq 0} \cos \left(\varphi-\varphi_{0}(\mathbf{r}, \varphi)\right) \mathrm{d} \mathbf{r}}{\int_{0}^{2 \pi} \int_{V_{F} / \cos \left(\varphi_{0}(\mathbf{r}, \varphi)-\varphi_{r}^{\prime}\right) \geq 0} \cos \left(\varphi-\varphi_{0}(\mathbf{r}, \varphi)\right) \mathrm{d} \mathbf{r} \mathrm{d} \varphi_{r}^{\prime}} .
$$


For a specular reflection law, the local bidirectional reflectivity is null at the local scale, except when the incident and reflected directions are associated through the Snell-Descartes law. This reflectivity is normalized with respect to reflecting directions and is then

$$
\rho_{\nu}^{\prime \prime} \text { spec }\left(\mathbf{u}, \mathbf{u}_{r}, \mathbf{n}\right)=\frac{\left[1-\frac{3}{2} \alpha_{\nu}^{h}(-\mathbf{u} . \mathbf{n})\right]}{(-\mathbf{u} . \mathbf{n})} \delta\left(\mathbf{u}_{r}^{\|}+\mathbf{u}^{\|}\right) \delta\left(\mathbf{u}_{r}^{\perp}-\mathbf{u}^{\perp}\right),
$$

where $\|$ denotes the component of a vector along $\mathbf{n}$, and ${ }^{\perp}$ its component perpendicular to $\mathbf{n}$. Taking into account Eqs. 19 and 21, $p_{\nu}^{\text {spec }}$ can be written at the global scale as

$$
\begin{aligned}
& p_{\nu}^{\text {spec }}\left(\theta, \varphi, \theta_{r}, \varphi_{r}\right)=4 \pi \delta\left(\cos \theta_{r}-\cos \theta\right) w_{\nu}\left(\theta, \varphi, \varphi_{r}\right), \\
& w_{\nu}\left(\theta, \varphi, \varphi_{r}\right)=\frac{\left[1-3 / 2 \alpha_{\nu}^{h} \sin \theta\left|\sin \left(\frac{\varphi_{r}-\varphi}{2}\right)\right|\right] j\left(\varphi, \varphi_{r}\right)}{\int_{0}^{2 \pi}\left[1-3 / 2 \alpha_{\nu}^{h} \sin \theta\left|\sin \left(\frac{\varphi_{r}^{\prime}-\varphi}{2}\right)\right|\right] j\left(\varphi, \varphi_{r}^{\prime}\right) \mathrm{d} \varphi_{r}^{\prime}} .
\end{aligned}
$$

In this expression, we have used Eq. 4 and the properties of the incidence and reflection angles of the Snell-Descartes law at the local scale, to write

$$
-\cos \left(\varphi-\varphi_{0}\right)=\cos \left(\varphi_{r}-\varphi_{0}\right)=\left|\cos \left(\frac{\varphi_{r}-\varphi \pm \pi}{2}\right)\right|=\left|\sin \left(\frac{\varphi_{r}-\varphi}{2}\right)\right| \geq 0
$$

In the $\theta=\pi / 2$ plane and for a given couple $\left(\varphi, \varphi_{r}\right), j\left(\varphi, \varphi_{r}\right)$ is the proportion of surface elements in the shooting area that give the impact points such as $\varphi_{r}$ is the specular reflection angle associated to $\varphi$.

All the results related to both diffuse and specular reflection laws are obtained by a Monte Carlo technique. Two examples of phase functions are plotted in Fig. .4.

\subsection{Effective medium optical index}

The effective scattering coefficient $\sigma_{\nu}(\theta, \varphi)$, equal to $\beta(\theta, \varphi)-\kappa_{\nu}(\theta, \varphi)$, has been determined independently of $p_{\nu}\left(\theta, \varphi, \theta_{r}, \varphi_{r}\right)$. Consequently, it is necessary to introduce an effective optical index $n_{\nu}(\theta, \varphi)$ in order to comply with the equilibrium solution of the Radiative Transfer Equation (RTE). Consequently, $n_{\nu}$ has to fulfill the two following conditions, as established in Appendices 2 and 3

$$
n_{\nu}^{2}\left(\theta_{r}, \varphi_{r}\right) \sigma_{\nu}\left(\theta_{r}, \varphi_{r}\right)=\frac{1}{4 \pi} \int_{4 \pi} n_{\nu}^{2}(\theta, \varphi) \sigma_{\nu}(\theta, \varphi) p_{\nu}\left(\theta, \varphi, \theta_{r}, \varphi_{r}\right) \mathrm{d} \Omega
$$




$$
\int_{4 \pi} \kappa_{\nu}(\theta, \varphi) n_{\nu}^{2}(\theta, \varphi) \mathrm{d} \Omega=A \pi \alpha_{\nu}^{h}
$$

in which all the quantities $\sigma_{\nu}, \kappa_{\nu}$ and $p_{\nu}$ have been previously determined.

In the case of a diffuse reflection law, it has been numerically established that $n^{2}$ is independent of $\nu$, and that the product $n^{2}(\theta, \varphi) \beta^{+}(\theta, \varphi)$ is equal to $\pi / 4$. Indeed, in any point of the transparent phase of an optically thin medium, the intensity at the local scale due to the wall emission, $\alpha_{\nu}^{h} I_{\nu}^{\circ}\left(T_{w}\right)$, is isotropic. Therefore, the emission term of the RTE, $n^{2}(\theta, \varphi) \beta(\theta, \varphi) \alpha_{\nu}^{h} I_{\nu}^{\circ}\left(T_{w}\right)$, has to be also isotropic. This result can be found again by identification of the expressions of the power emitted in the volume of a REV1 with the flux emitted by the walls in this REV1.

\subsection{First homogenization}

In conclusion, this first homogenization allows us to define all the radiative properties of an effective medium associated with a porous medium with an opaque solid phase. The radiative flux, in tensorial convention, is

$$
q_{i}^{R}\left(x_{k}\right)=\Pi \int_{0}^{\infty} \int_{4 \pi} I_{\nu}\left(x_{k}, \theta, \varphi\right) u_{i}(\theta, \varphi) \mathrm{d} \Omega \mathrm{d} \nu, \quad i=x, y, z
$$

where $I_{\nu}$ is the intensity of the effective medium within the transparent phase, as discussed in Ref. [6]. For any reflection law, the current intensity $I_{\nu}$ is obtained by solving the RTE

$$
\begin{aligned}
& u_{j}(\theta, \varphi) \frac{\partial I_{\nu}}{\partial x_{j}}\left(x_{k}, \theta, \varphi\right)+\beta(\theta, \varphi) I_{\nu}\left(x_{k}, \theta, \varphi\right) \\
= & \kappa_{\nu}(\theta, \varphi) n_{\nu}^{2}(\theta, \varphi) I_{\nu}^{\circ}\left[T_{w}\left(x_{k}\right)\right]+\frac{1}{4 \pi} \int_{4 \pi} \sigma_{\nu}\left(\theta^{\prime}, \varphi^{\prime}\right) p_{\nu}\left(\theta^{\prime}, \varphi^{\prime}, \theta, \varphi\right) I_{\nu}\left(x_{k}, \theta^{\prime}, \varphi^{\prime}\right) \mathrm{d} \Omega^{\prime}
\end{aligned}
$$

where the cartesian coordinates $x_{j}$ of a current point are introduced. 


\section{Radiative conductivity tensor}

\subsection{Perturbation method (general case)}

The purpose of this section is to develop, independently of the system geometry, the analytical expression of the radiative flux in the optically thick limit, in the case of effective radiative properties depending on both $\theta$ and $\varphi$. Under conditions defined in the following, the RTE can be treated by a perturbation method. We introduce the typical size $\delta$ of a second REV, called REV2, larger than REV1 but small in front of the global system size $L$, and the parameter

$$
\eta(\theta, \varphi)=\frac{1}{\beta(\theta, \varphi) \delta}
$$

Introducing the typical scale $\delta$ in the RTE (Eq. 27) leads to

$$
\begin{aligned}
& \eta(\theta, \varphi) u_{j}(\theta, \varphi) \frac{\partial I_{\nu}}{\partial x_{j}^{+}}\left(x_{k}^{+}, \theta, \varphi\right)+I_{\nu}\left(x_{k}^{+}, \theta, \varphi\right)=\frac{\kappa_{\nu}(\theta, \varphi)}{\beta(\theta, \varphi)} n_{\nu}^{2}\left(\theta_{r}, \varphi_{r}\right) I_{\nu}^{\circ}\left[T_{w}\left(x_{k}^{+}\right)\right] \\
& +\frac{1}{4 \pi \beta(\theta, \varphi)} \int_{4 \pi} \sigma_{\nu}\left(\theta^{\prime}, \varphi^{\prime}\right) p_{\nu}\left(\theta^{\prime}, \varphi^{\prime}, \theta, \varphi\right) I_{\nu}\left(x_{k}^{+}, \theta^{\prime}, \varphi^{\prime}\right) \mathrm{d} \Omega^{\prime}
\end{aligned}
$$

where $x_{j}^{+}$is equal to $x_{j} / \delta$. We now assume that the perturbation parameter $\eta(\theta, \varphi)$ is very small, and more precisely, that the REV2 absorption optical thickness is large for all considered frequencies, i.e.

$$
\frac{3}{\min \left(\kappa_{\nu}\right)}<\delta \ll L \quad \Longleftrightarrow \quad \operatorname{Kn}_{\nu}^{a}=\frac{1}{\kappa_{\nu}(\theta, \varphi) \delta}<\frac{1}{3}
$$

as discussed in Appendix 4 and Ref. [19]. The model validity strictly depends on the emission-absorption phenomena, even if the results will mainly depend on extinction. $\mathrm{Kn}_{\nu}^{a}$, defined by Eq. 30, is a radiative Knudsen number which plays the same role as the Knudsen number in the molecular conduction theory, also based on a perturbation method (see for instance, Ref. [20]).

We search a solution of Eq. 29 under the form $I_{\nu}=I_{\nu}^{(0)}+I_{\nu}^{(1)} . I_{\nu}^{(0)}$ is the zero order perturbation solution, also called Local Thermodynamical Equilibrium (LTE) solution, independent of $\eta$ and uniform within the REV2. $I_{\nu}^{(1)}$ is the first order perturbation solution, proportional to $\eta$, and consequently depending on spatial gradients at a scale $X_{k}$ larger than $\delta$. The temperature is then written $T_{w}\left(X_{k}\right)$.

As established in the end of Appendix 2, $I_{\nu}^{(0)}$ is equal to the equilibrium intensity $n_{\nu}^{2}(\theta, \varphi) I_{\nu}^{\circ}$. Consequently, $I_{\nu}^{(1)}$, the first order perturbation solution of Eq. 29, spatially uniform within the REV2 at location $X_{k}$, is obtained from 


$$
\begin{aligned}
I_{\nu}^{(1)}\left(X_{k}, \theta, \varphi\right) & =-\frac{u_{j}(\theta, \varphi) n_{\nu}^{2}(\theta, \varphi)}{\beta(\theta, \varphi)} \frac{\mathrm{d} I_{\nu}^{\circ}}{\mathrm{d} T_{w}}\left[T_{w}\left(X_{k}\right)\right] \frac{\partial T_{w}}{\partial X_{j}}\left(X_{k}\right) \\
& +\frac{1}{4 \pi \beta(\theta, \varphi)} \int_{4 \pi} \sigma_{\nu}\left(\theta^{\prime}, \varphi^{\prime}\right) p_{\nu}\left(\theta^{\prime}, \varphi^{\prime}, \theta, \varphi\right) I_{\nu}^{(1)}\left(X_{k}, \theta^{\prime}, \varphi^{\prime}\right) \mathrm{d} \Omega^{\prime}
\end{aligned}
$$

Without scattering $\left(\sigma_{\nu}=0\right)$, the first term of the right handside is the solution of the equation. Therefore, the scattering source term can be seen as a perturbation of this solution, which is calculated by an iterative technique for both diffuse and specular reflection laws, determined by Eqs. 9, 12, 14, 16 and 17. If we call $I_{\nu}^{n-s}$ the no scattering solution of Eq. 31, and $S_{\nu}$ the scattering operator (defined by the second term of the right handside of Eq. 31), the exact solution of this equation can be written under the form

$$
I_{\nu}^{(1)}=\sum_{k=0}^{+\infty} S_{\nu}^{k}\left(I_{\nu}^{n-s}\right)
$$

where the index $k$ means that the operator $S_{\nu}$ is applied $k$ times. A truncation of this infinite series is necessary for numerical calculation. We say that convergence is reached when the relative difference between the values at ranks $k$ and $k+1$ of the truncated partial sum is smaller than a given percentage. For instance, in the case of a diffuse reflection law in the squared configuration, with $\alpha_{\nu}^{h}=\alpha^{h}=2 / 9,7$ iterations are necessary to obtain converged values for every couple $(\theta, \varphi)$ when the relative difference is of $1 \%$. This number is growing to 30 iterations for a relative difference of $10^{-9} \%$. For any configuration, reflection law and value of $\alpha^{h}$, no significant evolution of the radiative conductivities is observed under a precision of $10^{-2} \%$, and this value is then chosen in the following. These calculations last never more than a few hours on a current single processor computer. Finally, after reaching convergence, we obtain an approximate expression of the first order intensity $I_{\nu}^{(1)}$, which is always proportional to $-\partial T_{w} / \partial X_{j}$, whatever the truncation rank is.

The contribution of $I_{\nu}^{(0)}$ to the radiative flux given by Eq. 26 is null, as established in Appendix 2. The only contribution comes from the first order intensity $I_{\nu}^{(1)}$, solution of the implicit Eq. 31. Taken into account Eqs. 26 and $32, q_{i}^{R}$ can be written under the form

$$
q_{i}^{R}\left(X_{k}\right)=-\frac{\partial T_{w}}{\partial X_{j}}\left(X_{k}\right) \Pi \frac{\pi}{A} \int_{0}^{\infty} \frac{\mathrm{d} I_{\nu}^{\circ}}{\mathrm{d} T_{w}}\left[T_{w}\left(X_{k}\right)\right] \int_{4 \pi} u_{i}(\theta, \varphi) Y_{j}\left(\Pi, \alpha_{\nu}^{h}, \theta, \varphi\right) \mathrm{d} \Omega \mathrm{d} \nu,(33)
$$

where $Y_{j}$ is a vector issued from the $I_{\nu}^{(1)}$ truncated solution, obtained at the last iteration. Finally, we directly find a radiative Fourier law, also called 
Rosseland approximation, i.e.

$$
q_{i}^{R}=-k_{i j} \frac{\partial T_{w}}{\partial X_{j}}
$$

where $k_{i j}$ is given by

$$
k_{i j}\left[\Pi, A, \alpha_{\nu}^{h}, T_{w}\left(X_{k}\right)\right]=\Pi \frac{\pi}{A} \int_{0}^{\infty} \frac{\mathrm{d} I_{\nu}^{\circ}}{\mathrm{d} T_{w}}\left[T_{w}\left(X_{k}\right)\right] k_{i j}^{+}\left(\Pi, \alpha_{\nu}^{h}\right) \mathrm{d} \nu \quad i, j=x, y, z .(35
$$

In practice, $k_{i j}^{+}\left(\Pi, \alpha_{\nu}^{h}\right)$, equal to $\int_{4 \pi} u_{i}(\theta, \varphi) Y_{j}\left(\theta, \varphi, \Pi, \alpha_{\nu}^{h}\right) \mathrm{d} \Omega$, is tabulated. It is worth of notice that the spatial scale in use for practical application with this homogenization is $X_{j}$, of smallest resolution $\delta$.

Under the approximation of gray walls $\left(\alpha_{\nu}^{h}=\alpha^{h}\right)$, we simply obtain

$$
k_{i j}\left[\Pi, A, \alpha^{h}, T_{w}\left(X_{k}\right)\right]=\frac{\Pi}{A} 4 \sigma T_{w}\left(X_{k}\right)^{3} k_{i j}^{+}\left(\Pi, \alpha^{h}\right) .
$$

The properties of the radiative conductivity tensor $k_{i j}$ depend on the considered porous medium statistical symmetries. For a statistically isotropic medium, $k_{i j}$ degenerates in a simple scalar radiative conductivity $k$.

\subsection{Examples of Results (rod bundles)}

In the case of rod bundles, for both specular and diffuse reflection laws, the radiative conductivity model cannot be applied for the infinite direction. The validity criterion of Eq. 30 is never verified. Morever, the directional integrals in Eq. 26 do not converge.

In the following, we only consider a two dimensional application characterized by uniform fields of any quantity along $z$ axis. We obtain : $k_{x x}^{+}=k_{y y}^{+}=k^{+}$and $k_{x y}^{+}, k_{y x}^{+}$are null, for symmetry reasons. These results have been numerically checked with a high accuracy. The effective medium is then characterized, for 2D applications, by scalar conductivities $k$ for both triangular and squared configurations, and both diffuse and specular reflection laws. $k$ has only been calculated up to $\Pi=0.75$, for reasons detailed in the following. Results have been fitted by $a(\Pi) \alpha^{h^{2}}+b(\Pi) \alpha^{h}+c(\Pi)$. There is an exception for $\Pi=0.2146$ with a specular reflection law in the squared configuration, where the fit is a line, due to discretization approximations. The highest relative error introduced by the fit for all $\Pi$ and $\alpha^{h}$ values is less than $3.10^{-3}$ in the diffuse case and less than $2 \cdot 10^{-2}$ in the specular case for the two configurations. Coefficients $a, b$ and $c$ are given in Table 3.2 .

As pointed out in the introduction, there is a lack of accurate experiments that 
could validate this radiative conductivity model. Nevertheless, some theoretical models have been proposed in previous works, but for a diffuse reflection law only. In a squared configuration with $\Pi=0.5353$ and $\alpha^{h}=0.8$, the calculations of Manteufel [14] give $k^{+}=6.52$, the ones of Fischer [9] $k^{+}=4.33$, and our model $k^{+}=10.15$. In a triangular configuration with $\Pi=0.5373$ and $\alpha^{h}=0.8$, the calculations of Manteufel lead to $k^{+}=5.19$ while our model gives $k^{+}=4.95$. In both configurations, the order of magnitude is similar, but we obtain significantly higher values of radiative conductivity in the squared case.

It is of practical interest to discuss the influence of the dependence of $\beta$ on $\varphi$ in the radiative conductivity calculation. In order to eliminate the influence of the scattering source term, we have chosen $\alpha^{h}=1$, which corresponds to non reflecting rods. Comparisons between values of $k$ obtained from the extinction coefficient $\beta(\pi / 2, \varphi)$, associated with $G_{e}(s, \pi / 2, \varphi)$ and the radiative conductivity obtained from the $\varphi$ independent extinction coefficient $\beta(\pi / 2)$ associated with $G_{e}(s, \pi / 2)$, have been carried out. It is worth of notice that, for conductivity calculations, $\beta$ can be considered as independent of $\varphi$ for $\Pi<0.5$ with a relative error on conductivity less than 0.03 . Consequently, as $\beta(\pi / 2)$ is accurately determined for $\Pi<0.5$ (see Fig. 3(b)), $k$ is determined with an accuracy of a few percent from the smallest values of $\Pi$ to 0.5 , but the uncertainty on $k$ increases when $\Pi$ increases. For this reason, we have only and arbitrarily calculated $k$ up to $\Pi=0.75$, associated with a relative error on $\beta$ of about 0.1 .

\section{Conclusion}

In a first part, statistically strongly anisotropic porous media (rod bundles), have been modeled by effective semi transparent media, from the Radiative Distribution Function Identification method. Directional extinction, absorption and scattering coefficients and a bidirectional scattering phase function have been determined with a quantitative validity criterion. As the RDFI method is accurate, it has been established that for rod bundles: i) the Beer's law in use in the effective medium is a rather accurate model for low porosity (less than 0.5); in these conditions, extinction and absorption coefficients can be considered in practice as depending only on the angle with the rod axis (vertical-based azimuth); ii) the Beer's law is less and less accurate when the porosity increases. An advantage of the RDFI approach is to clearly establish this type of limitation. Consequently, an important research topic for many applications, in nuclear safety for instance, is to develop original models for porous media where the Beer's laws are not valid.

In a second part, for optically thick media and when the Beer's law validity criterion is reasonably verified, a radiative conductivity tensor has been di- 
rectly determined from the whole set of effective radiative properties, including strongly anisotropic bidirectional scattering phase function. A key point is the validity criterion of this model, based on the absorption optical thickness, even if the radiative conductivity also strongly depends on the scattering coefficient and phase function.

\section{References}

[1] T. Hendricks, J. Howell, Inverse radiative analysis to determine spectral radiative properties using the discrete ordinates method, International Journal of Heat and Mass Transfer 2 (1994) 75-80.

[2] T. Hendricks, J. Howell, New radiative analysis approach for reticulated porous ceramics using discrete ordinates method, ASME Journal of Heat Transfer 4 (118) (1996) 911-917.

[3] T. J. Hendricks, J. R. Howell, Absorption/scattering coefficients and scattering phase function in reticulated porous ceramics, ASME Journal of Heat Transfer. 118 (1) (1996) 79-87.

[4] D. Baillis, J. Sacadura, Identification of spectral radiative properties of polyurethane foam from hemispherical and bi-directional transmittance and reflectance measurement, Journal of Quantitative Spectroscopy and Radiative Transfer 73 (2002) 297-306.

[5] B. P. Singh, M. Kaviany, Modelling radiative heat transfer in packed beds, International Journal of Heat and Mass Transfer 6 (35) (1992) 1397-1405.

[6] M. Tancrez, J. Taine, Direct identification of absorption and scattering coefficients and phase function of a porous medium by a Monte Carlo technique, International Journal of Heat and Mass Transfer 47 (2004) 373-383.

[7] B. Zeghondy, E. Iacona, J. Taine, Determination of the anisotropic radiative properties of a porous material by radiative distribution function identification (RDFI), International Journal of Heat and Mass Transfer 49 (2006) 2810-2819.

[8] J. Petrasch, P. Wyss, A. Steinfeld, Tomography-based Monte Carlo determination of radiative properties of reticulated porous ceramics, Journal of Quantitative Spectroscopy and Radiative Transfer 105 (2007) 180-197.

[9] L. Fischer, Spent fuel heating analysis code for consolidated and unconsolidated fuel, in: Proceedings of the $9^{\text {th }}$ international Symposium on Packaging and Transportation of radioactive Materials (PATRAM) '89, Oak Ridge National laboratory, 1989.

[10] R. Cox, Radiative heat transfer in arrays of parallel cylinders, Ph.D. thesis, University of Tennessee, Knoxville, USA (December 1976). 
[11] C. Tien, R. Sanchez, D. Mandell, C. McDaniel, Surface radiative exchange in rod bundles, ASME Journal of Heat Transfer 101 (1979) 378.

[12] M. Sohal, A radiation heat transfer model for the SCDAP code, Nuclear Technology 75 (1986) 196-204.

[13] P. Chatelard, ICARE/CATHARE a computer code for analysis of severe accidents in LWR's, ICARE v3 mod1.1, Tech. rep., IPSN (2001).

[14] R. Manteufel, Heat transfer in an enclosed rod array, Ph.D. thesis, Departement of Mechanical Engineering, Massachusetts Institute of Technology, USA (May 1991).

[15] H. Hottel, A. Sarofim, Radiative transfer, McGraw-Hill Book Company, New York, 1967.

[16] G. Mazza, C. Berto, G. Barreto, Evaluation of radiative heat transfer properties in dense particulate media, Powder Technology 67 (1991) 137-144.

[17] P. Rubiolo, J. Gatt, Modeling of the radiative contribution to heat transfer in porous media composed of spheres or cylinders, International Journal of Thermal Sciences 41 (2002) 401-411.

[18] E. Chalopin, Caractérisation des propriétés radiatives d'un milieu poreux avec une méthode RDFI. Application à un cœur de réacteur à eau sous pression durant un accident grave., Thèse de doctorat, École Centrale Paris, France (2008).

[19] J. Taine, E. Iacona, J. Petit, Transferts Thermiques, $4^{\text {th }}$ Edition, Dunod, 2008.

[20] S. Chapmann, T. Cooling, The Mathematical Theory of Non Uniform Gases, Cambridge University Press, 1970.

\section{Appendices}

\section{Appendix 1}

This Appendix deals with the dependence on $\theta$ of $G_{e}$ and $P_{a \nu}$ for rod bundles (Sec. 2.1). From Eq. 6, we obtain, using the transformation $s^{\prime}=s^{\prime \prime} / \sin \theta$

$$
\begin{aligned}
& G_{e}(s, \theta, \varphi) \\
= & \int_{0}^{s \sin \theta} \frac{1}{V_{F}} \int_{V_{F}} \delta\left[\frac{s^{\prime \prime}}{\sin \theta}-\frac{s_{0}(\mathbf{r}, \pi / 2, \varphi)}{\sin \theta}\right] \mathrm{d} \mathbf{r} \frac{\mathrm{d} s^{\prime \prime}}{\sin \theta} \\
= & \int_{0}^{s \sin \theta} \frac{1}{V_{F}} \int_{V_{F}} \delta\left[s^{\prime \prime}-s_{0}(\mathbf{r}, \pi / 2, \varphi)\right] \mathrm{d} \mathbf{r} \mathrm{d} s^{\prime \prime} \\
= & G_{e}(s \sin \theta, \pi / 2, \varphi) .
\end{aligned}
$$


With the same transformation, in the case of a specular reflection law, $P_{a \nu}^{\operatorname{spec}}(s, \theta, \varphi)$ given by Eq. 11, becomes, using Eq. 4

$$
\begin{aligned}
& P_{a \nu}^{\text {spec }}(s, \theta, \varphi) \\
= & -\frac{3}{2} \alpha_{\nu}^{h} \sin \theta \int_{0}^{s \sin \theta} \frac{1}{V_{F}} \int_{V_{F}} \cos \left[\varphi-\varphi_{0}(\mathbf{r}, \varphi)\right] \delta\left[\frac{s^{\prime \prime}}{\sin \theta}-\frac{s_{0}(\mathbf{r}, \pi / 2, \varphi)}{\sin \theta}\right] \mathrm{d} \mathbf{r} \frac{\mathrm{d} s^{\prime \prime}}{\sin \theta} \\
= & \sin \theta \int_{0}^{s \sin \theta} \frac{1}{V_{F}} \int_{V_{F}}-\frac{3}{2} \alpha_{\nu}^{h} \cos \left[\varphi-\varphi_{0}(\mathbf{r}, \varphi)\right] \delta\left[s^{\prime \prime}-s_{0}(\mathbf{r}, \pi / 2, \varphi)\right] \mathrm{d} \mathbf{r} \mathrm{d} s^{\prime \prime} \\
= & \sin \theta P_{a \nu}^{s p e c}(s \sin \theta, \pi / 2, \varphi) .
\end{aligned}
$$

Appendix 2

This appendix deals with some properties at equilibrium or in LTE conditions. When the effective medium is at equilibrium, characterized by $n_{\nu}^{2}(\theta, \varphi) I_{\nu}^{\circ}\left(T_{w}\right)$, the RTE becomes

$$
\begin{aligned}
\beta(\theta, \varphi) n_{\nu}^{2}(\theta, \varphi) I_{\nu}^{\circ}\left(T_{w}\right) & =\kappa_{\nu}(\theta, \varphi) n_{\nu}^{2}(\theta, \varphi) I_{\nu}^{\circ}\left(T_{w}\right) \\
& +\frac{I_{\nu}^{\circ}\left(T_{w}\right)}{4 \pi} \int_{0}^{4 \pi} \sigma_{\nu}\left(\theta^{\prime}, \varphi^{\prime}\right) p_{\nu}\left(\theta^{\prime}, \varphi^{\prime}, \theta, \varphi\right) n_{\nu}^{2}\left(\theta^{\prime}, \varphi^{\prime}\right) \mathrm{d} \Omega^{\prime} .
\end{aligned}
$$

Consequently, we obtain:

$$
\sigma_{\nu}(\theta, \varphi) n_{\nu}^{2}(\theta, \varphi)=\frac{1}{4 \pi} \int_{0}^{4 \pi} \sigma_{\nu}\left(\theta^{\prime}, \varphi^{\prime}\right) p_{\nu}\left(\theta^{\prime}, \varphi^{\prime}, \theta, \varphi\right) n_{\nu}^{2}\left(\theta^{\prime}, \varphi^{\prime}\right) \mathrm{d} \Omega^{\prime} .
$$

At this step, we search $I_{\nu}^{(0)}$, the zero order perturbation solution of Eq. 29. The first term of the first member of this equation, proportional to $\eta$, vanishes and we obtain

$$
\begin{aligned}
\beta(\theta, \varphi) I_{\nu}^{(0)}\left(X_{k}, \theta, \varphi\right) & =\kappa_{\nu}(\theta, \varphi) n_{\nu}^{2}(\theta, \varphi) I_{\nu}^{\circ}\left[T_{w}\left(X_{k}\right)\right] \\
& +\frac{1}{4 \pi} \int_{0}^{4 \pi} \sigma_{\nu}\left(\theta^{\prime}, \varphi^{\prime}\right) p_{\nu}\left(\theta^{\prime}, \varphi^{\prime}, \theta, \varphi\right) I_{\nu}^{(0)}\left(X_{k}, \theta^{\prime}, \varphi^{\prime}\right) \mathrm{d} \Omega^{\prime}
\end{aligned}
$$

By taking into account Eqs. .2 and .3, we obtain $I_{\nu}^{(0)}\left(X_{k}, \theta, \varphi\right)=n_{\nu}^{2}(\theta, \varphi) I_{\nu}^{\circ}\left[T_{w}\left(X_{k}\right)\right]$ as the unique solution.

Appendix 3 
This appendix deals with the normalization of $n_{\nu}^{2}(\theta, \varphi)$. At the limit of an optically thin medium, we identify in $\mathrm{d} \nu$ the flux emitted by the volume of the effective fluid phase contained in a REV1, $\mathrm{d} V_{F}$, with the flux emitted by the real interfacial area $\mathrm{d} S$ in the same REV1

$$
\mathrm{d} V_{F} \mathrm{~d} \nu \int_{4 \pi} \kappa_{\nu}(\theta, \varphi) n_{\nu}^{2}(\theta, \varphi) I_{\nu}^{\circ}\left(T_{w}\right) \mathrm{d} \Omega=\mathrm{d} S \mathrm{~d} \nu \int_{2 \pi} \alpha_{\nu}^{\prime}(\theta, \varphi) I_{\nu}^{\circ}\left(T_{w}\right) \cos \theta \mathrm{d} \Omega .
$$

The specific area by unit volume of the transparent phase, $A$, is equal to $\mathrm{d} S / \mathrm{d} V_{F}$, and the unknown factor that normalizes $n_{\nu}^{2}(\theta, \varphi)$ is then given by

$$
\int_{4 \pi} \kappa_{\nu}(\theta, \varphi) n_{\nu}^{2}(\theta, \varphi) \mathrm{d} \Omega=A \pi \alpha_{\nu}^{h}
$$

Appendix 4

In this appendix, we define a criterion of validity for the radiative conduction model, in a similar way as the classical diffusion model for a material system.

Let us first precise the pertinent scale of a REV2, such as the perturbation method can be applied at this scale. Eq. 31 is identical to Eq. .3 of Appendix 2 after substitution of $\left(u_{j} / \beta\right) n_{\nu}^{2}\left(\mathrm{~d} I_{\nu}^{\circ} / \mathrm{d} T_{w}\right)\left(\partial T_{w} / \partial X_{j}\right)$ by $\left(\kappa_{\nu} / \beta\right) n_{\nu}^{2} I_{\nu}^{\circ}\left(T_{w}\right)$. A practical criterion is issued from the exponential features of the RTE solution: $\left(u_{j} / \beta\right) n_{\nu}^{2}\left(\mathrm{~d} I_{\nu}^{\circ} / \mathrm{d} T_{w}\right)\left(\partial T_{w} / \partial X_{j}\right)$ has to be 3 to 5 times smaller than $\left(\kappa_{\nu} / \beta\right) n_{\nu}^{2} I_{\nu}^{\circ}\left(T_{w}\right)$.

A practical formulation in non dimensional quantities is, for all considered frequencies,

$$
\kappa_{\nu} \delta>3 \text { to } 5, \quad \Longleftrightarrow \quad \mathrm{Kn}_{\nu}^{a}=\frac{1}{\kappa_{\nu} \delta}<\frac{1}{5} \text { to } \frac{1}{3},
$$

where $\mathrm{Kn}_{\nu}^{a}$ is the radiative Knudsen number. The model is valid if: i) the temperature field and all the medium radiative properties can be assumed uniform within the REV2 of size $\delta$ (or each phase of the REV2) for calculations at the zero order of perturbation, ii) the temperature gradient can be considered uniform in this same REV2 (or each phase within the REV2) for calculations at the first order of perturbation.

[Table 1 about here.]

[Fig. 1 about here.]

[Fig. 2 about here.] 
[Fig. 3 about here.]

[Fig. 4 about here.] 


\section{List of Figures}

.1 a) Triangular and b) squared rod bundle configurations, delimited by bold lines, and c) system of coordinates.

$.2 \quad$ For a triangular pattern and $\Pi=0.513$, extinction cumulated distribution functions $G_{e}(s, \pi / 2, \varphi)$ for different values of $\varphi\left(1.5^{\circ} \square, 7.5^{\circ}+, 13.5^{\circ} \circ, 22.5^{\circ} \times, 28.5^{\circ} \triangle\right)$, extinction cumulated distribution function $G_{e}(s, \pi / 2)$ for all values of $\varphi$ (thick line), and the corresponding adjusted semi transparent extinction cumulated distribution function $g_{e}(s, \pi / 2)$ (thin line). For the plots, the non dimensional abscissa $\beta s$ is used, where $\beta$ is the extinction coefficient identified from $G_{e}(s, \pi / 2)$.

.3 a) Extinction coefficient $\beta^{+}(\pi / 2, \varphi)$ and corresponding identification error for a triangular pattern, for different values of $\Pi(0.300+, 0.513 \boldsymbol{\Delta}, 0.850 \times, 0.990 \circ), \mathrm{b})$ extinction coefficient $\beta^{+}(\pi / 2)$ associated with $G_{e}(s, \pi / 2)$ versus $\Pi$ ( $\boldsymbol{\Delta}$ triangular, $\times$ squared pattern) and corresponding identification error ( $\triangle$ triangular, $\square$ squared pattern), and c) absorption coefficient $\kappa^{+}(\pi / 2)$ associated with $P_{a \nu}(s, \pi / 2)$ versus $\Pi(\boldsymbol{\Delta}$ triangular, $\times$ squared pattern $)$ and corresponding identification error ( $\triangle$ triangular, $\square$ squared pattern).

$.4 \quad$ For $\Pi=0.513$, a) $p^{\text {diff }}$ versus $\varphi_{r}-\varphi$, for different values of $\varphi$, b) $p^{\text {spec }}$ versus $\varphi_{r}-\varphi$, for $\alpha^{h}=0.8$ and $\theta=\pi / 2$, and for different values of $\varphi\left(0.5^{\circ}\right.$ thick solid line, $7.5^{\circ}$ thin solid line, $14.5^{\circ}$ thick dotted line, $21.5^{\circ}$ thin dotted line, $28.5^{\circ}$ mixted line). 


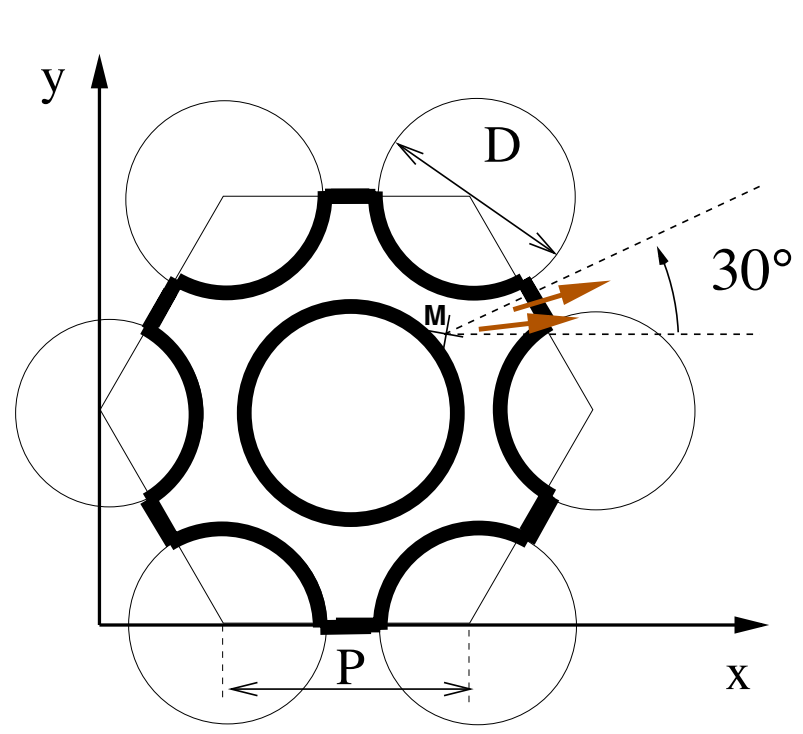

(a)

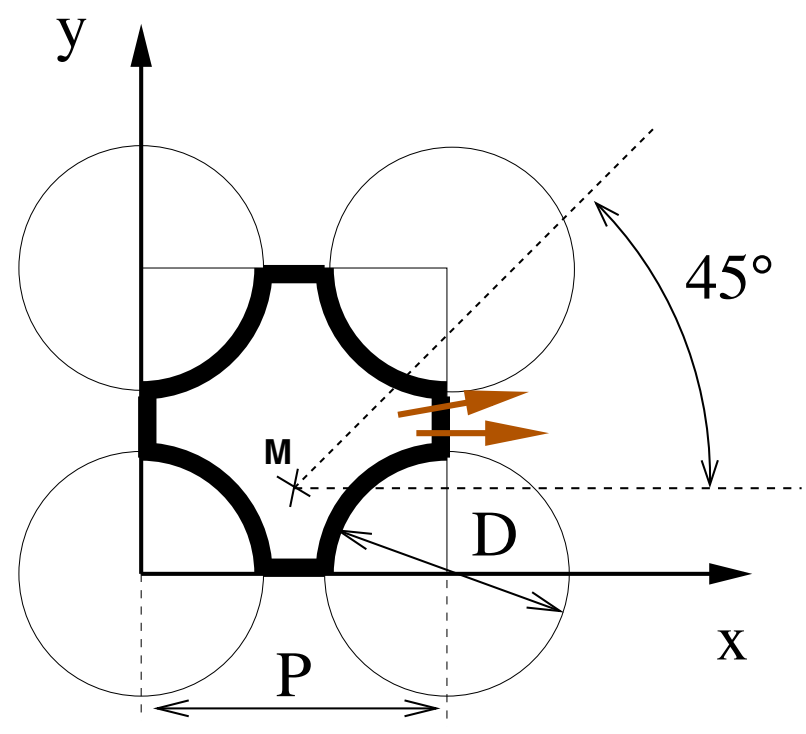

(b)

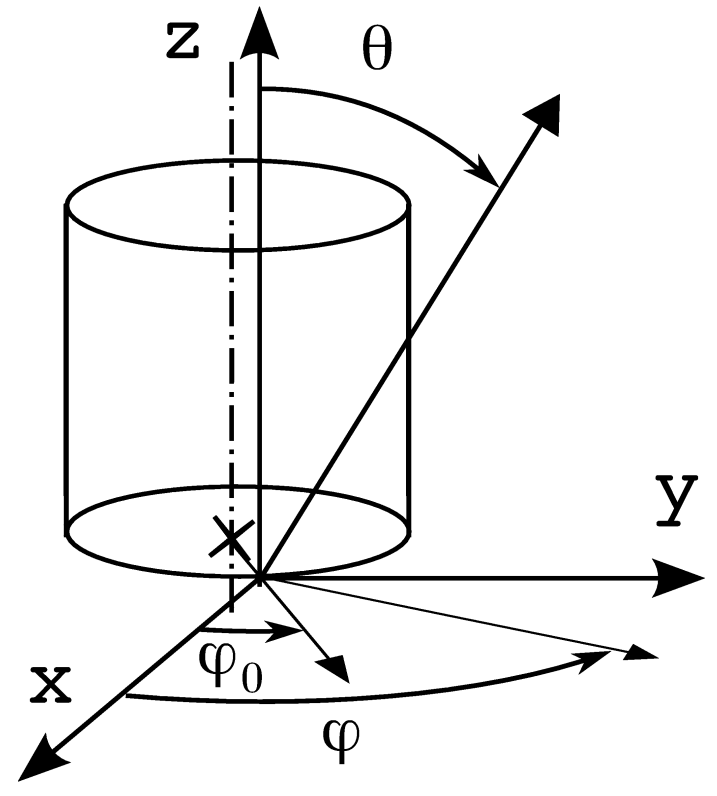

(c) 


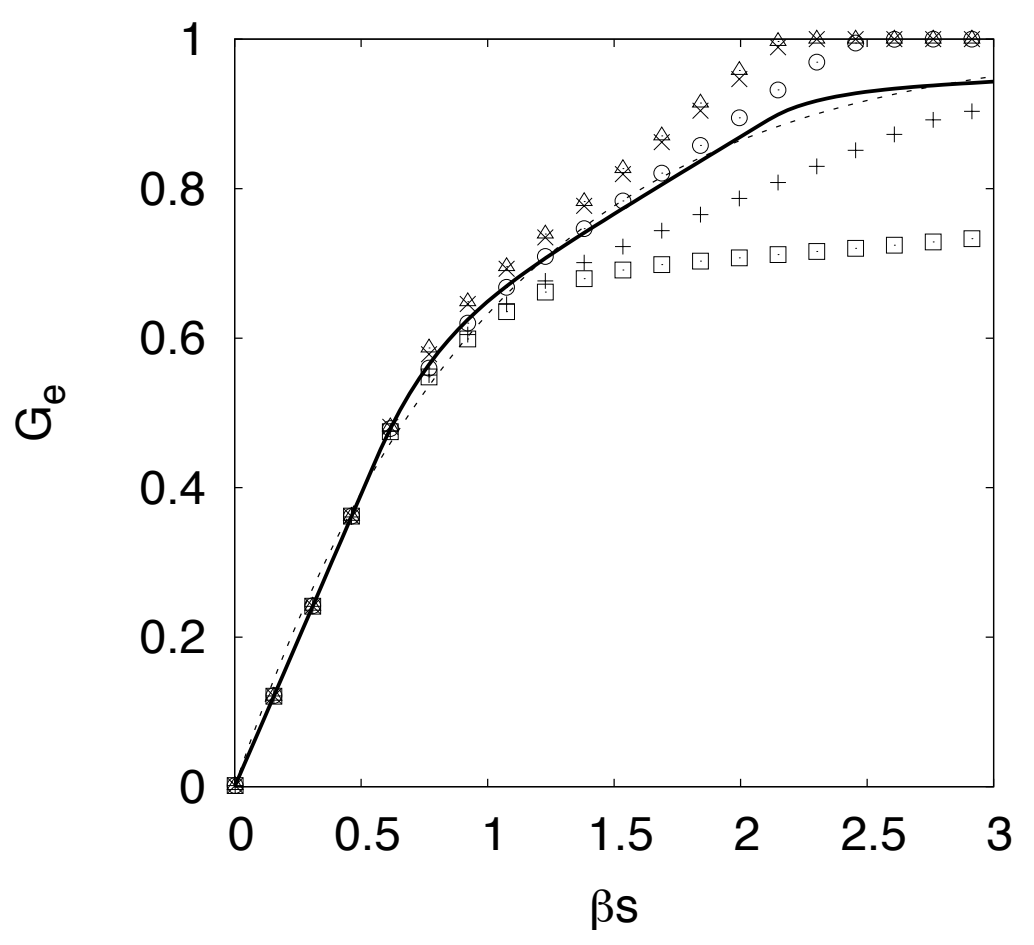



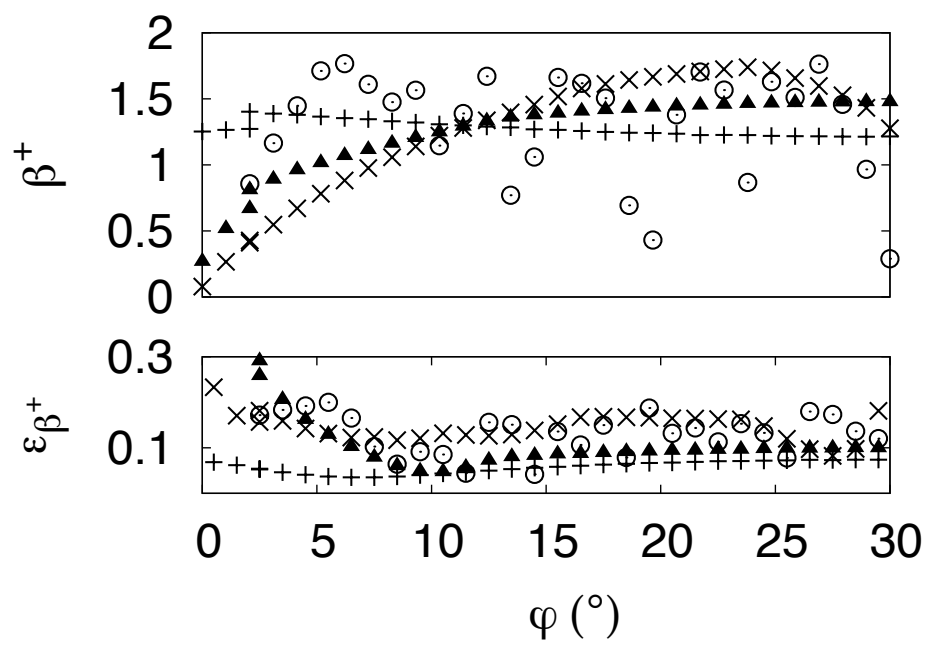

(a)

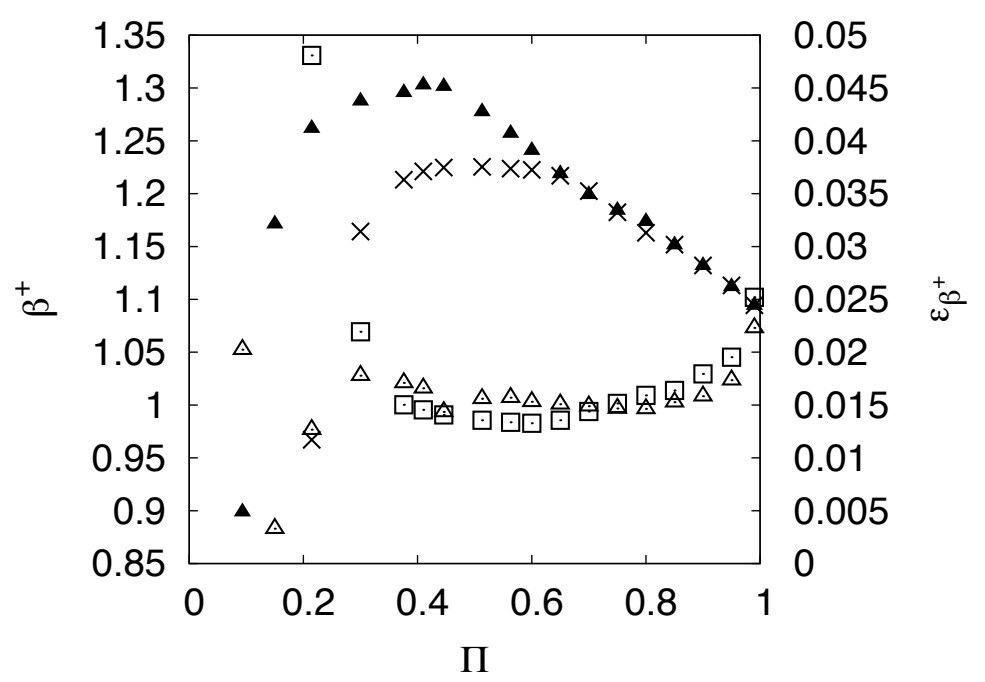

(b)

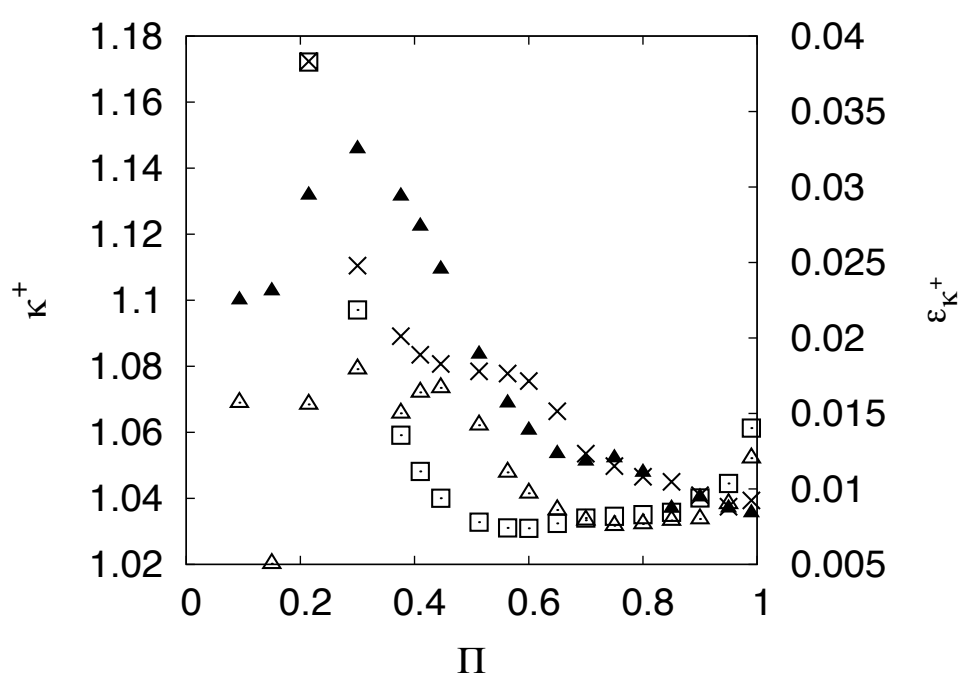

(c) 


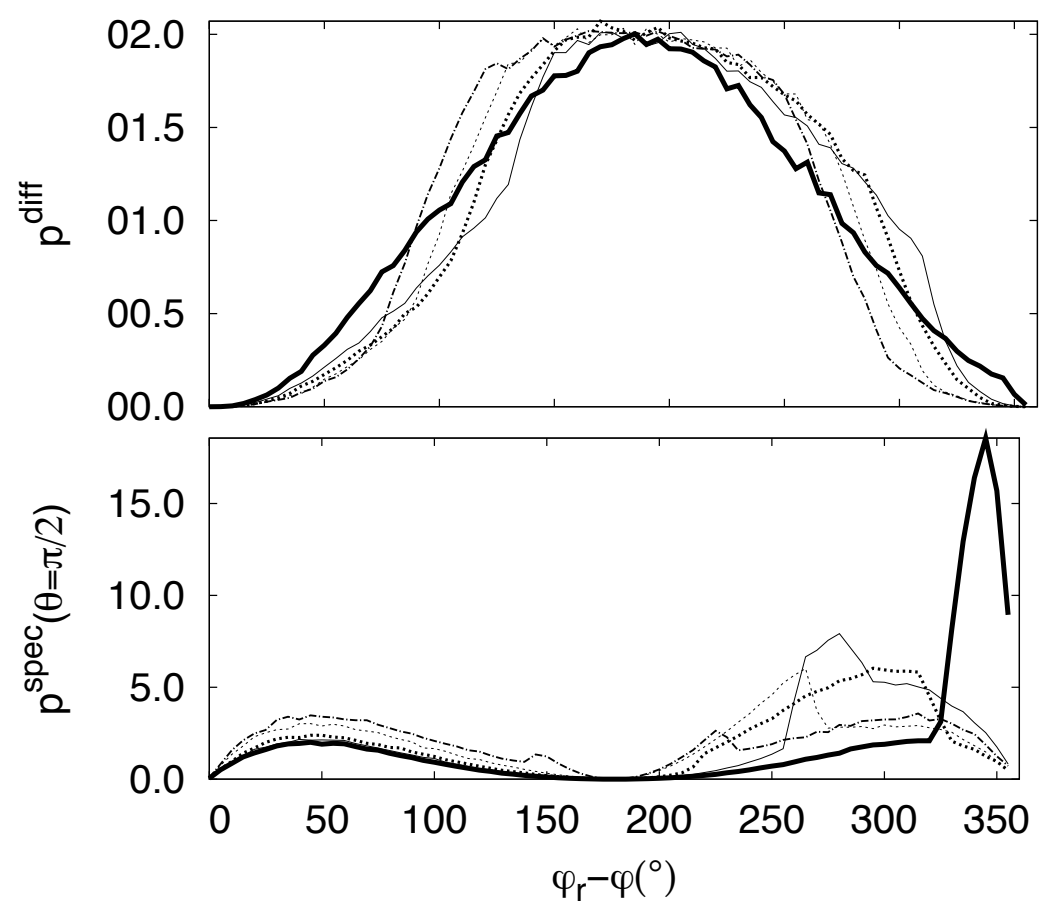




\section{List of Tables}

.1 Coefficients $a, b$ and $c$ versus $\Pi$ (from 0.093 to 0.75 ), for diffuse and specular reflection laws, and for triangular and squared configurations (NP stands for geometrically impossible porosities). 


\begin{tabular}{|c|c|c|c|c|c|c|c|c|c|c|c|c|}
\hline & \multicolumn{6}{|c|}{ Diffuse reflection } & \multicolumn{5}{c|}{ Specular reflection } \\
\hline \multirow{2}{*}{$\Pi$} & \multicolumn{3}{|c}{ Triangular } & \multicolumn{3}{c|}{ Squared } & \multicolumn{3}{c|}{ Triangular } & \multicolumn{3}{c|}{ Squared } \\
\hline 0.0930 & 0.93 & 1.14 & 4.06 & $\mathrm{NP}$ & $\mathrm{NP}$ & $\mathrm{NP}$ & 0.25 & 1.42 & 5.83 & $\mathrm{NP}$ & $\mathrm{NP}$ & $\mathrm{NP}$ \\
0.1500 & 0.73 & 0.88 & 3.13 & $\mathrm{NP}$ & $\mathrm{NP}$ & $\mathrm{NP}$ & 0.32 & 1.07 & 4.41 & $\mathrm{NP}$ & $\mathrm{NP}$ & $\mathrm{NP}$ \\
0.2146 & 0.49 & 0.58 & 2.08 & 0.78 & 1.07 & 3.48 & 0.40 & 0.68 & 2.80 & Line & Line & Line \\
0.3000 & 0.47 & 0.55 & 1.98 & 0.57 & 0.76 & 2.68 & 0.07 & 0.74 & 2.58 & -3.83 & 1.88 & 3.60 \\
0.3760 & 0.46 & 0.54 & 1.95 & 0.61 & 0.80 & 3.41 & -0.62 & 0.91 & 2.57 & -4.44 & 1.17 & 4.56 \\
0.4100 & 0.46 & 0.54 & 1.97 & 0.63 & 0.81 & 3.74 & -1.06 & 1.01 & 2.64 & -4.71 & 0.86 & 4.99 \\
0.4460 & 0.46 & 0.56 & 2.07 & 0.68 & 0.88 & 5.13 & -1.65 & 1.11 & 2.80 & -5.46 & -0.32 & 6.66 \\
0.5130 & 0.53 & 0.64 & 2.73 & 0.79 & 1.01 & 7.71 & -3.46 & 1.20 & 3.73 & -6.85 & -2.52 & 9.76 \\
0.5630 & 0.65 & 0.81 & 5.34 & 0.86 & 1.10 & 10.13 & -6.74 & -0.03 & 6.96 & -8.75 & -4.12 & 12.46 \\
0.6000 & 0.75 & 0.93 & 7.26 & 0.90 & 1.16 & 11.92 & -9.17 & -0.95 & 9.36 & -10.70 & -4.98 & 14.40 \\
0.6500 & 0.89 & 1.12 & 11.55 & 0.94 & 1.22 & 14.20 & -12.67 & -3.82 & 14.40 & -14.05 & -5.55 & 16.98 \\
0.7000 & 1.01 & 1.29 & 16.27 & 0.98 & 1.29 & 16.48 & -15.59 & -7.62 & 19.99 & -17.41 & -6.12 & 19.57 \\
0.7500 & 1.12 & 1.44 & 21.22 & 1.02 & 1.35 & 18.77 & -19.30 & -10.96 & 25.45 & -20.77 & -6.68 & 22.15 \\
\hline
\end{tabular}

